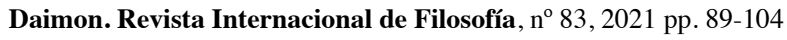

ISSN: 1130-0507 (papel) y 1989-4651 (electrónico)

http://dx.doi.org/10.6018/daimon.366051

Las obras se publican en la edición electrónica de la revista bajo una licencia Creative Commons ReconocimientoNoComercial-SinObraDerivada 3.0 España (texto legal). Se pueden copiar, usar, difundir, transmitir y exponer públicamente, siempre que: i) se cite la autoría y la fuente original de su publicación (revista, editorial y URL de la obra); ii) no se usen para fines comerciales; iii) se mencione la existencia y especificaciones de esta licencia de uso. (c) (1)(8) $(-)$

\title{
La exclusión social y la incorporación adversa. Hacia una crítica de un mundo en globalización*
}

\section{Social exclusion and adverse incorporation. Towards a critique of a globalizing world}

FRANCISCO BLANCO BROTONS ${ }^{* *}$

\begin{abstract}
Resumen: El objetivo de este artículo es someter a crítica el discurso de la exclusión social. Comenzaremos exponiendo algunos rasgos centrales de nuestro mundo en globalización para, a continuación, exponer las razones que hacen a este discurso profundamente inadecuado. Distrae la atención de características fundamentales de este mundo, de modo que puede ser instrumentalizado para ocultar relaciones que se encuentran a la base de la explotación, la dominación o la subordinación. Frente a este discurso se propondrá otro que podría evitar estos peligros, el de la incorporación adversa.
\end{abstract}

Palabras clave: exclusión, inclusión, globalización, explotación, incorporación adversa.

\begin{abstract}
The aim of this article is to criticize the discourse of social exclusion. I explain some key features of our globalizing world and why this discourse is profoundly inadequate to face them. It distracts attention from fundamental characteristics of our contemporary world, so that it can be used to obscure relationships at the root of exploitation, domination or subordination. Against this discourse, I propose the concept of adverse incorporation to avoid these dangers.
\end{abstract}

Keywords: exclusion, inclusion, globalization, exploitation, adverse incorporation.

Los conceptos y discursos mediante los que enmarcamos el mundo son piezas decisivas para toda filosofía crítica que pretenda no sólo hacer un análisis agudo de la realidad, sino especialmente cambiar las cosas. Para comprender una realidad abrumadoramente compleja, el primer paso de la humanidad siempre ha sido reducir la complejidad proyectando marcos conceptuales. Frente a esto, sin embargo, la tarea fundamental es "determinar qué

Recibido: 05/03/2019. Aceptado: 20/08/2019.

* Este artículo se ha elaborado en el marco de un proyecto de investigación financiado por el Ministerio de Economía y Competitividad: «Derechos humanos y justicia global en el contexto de las migraciones internacionales» (FFI2013-42521-P).

** Investigador en formación en el Instituto de Filosofía del Consejo Superior de Investigaciones Científicas. Correo electrónico: franciscojose.blanco@cchs.csic.es. Líneas de investigación: teorías de la justicia, justicia global, teorías de la democracia, globalización, ontología social. Publicaciones recientes: (2019) "Expanding the Idea of Structural Injustice: Migrants and Global Justice", en J. C. Velasco y M. C. La Barbera (Eds.), Challenging the Borders of Justice in the Age of Migrations (pp.117-138), Cham, Springer; (2019) "Sujeto y mundo. Premisas ontológicas del concepto kantiano de autonomía de la razón”, Pensamiento, 75 (284), 603-620. 
reducciones de complejidad son más adecuadas al mundo contemporáneo y cuales dejan fuera demasiados tonos y voces" (Wimmer y Schiller, 2002, 235). Si queremos herramientas conceptuales capaces de transformar el mundo, ellas deben estar bien ajustadas a nuestra realidad contemporánea, reduciendo la complejidad, pero no a costa de cercenar sus rasgos fundamentales. Los discursos son productos históricos, herramientas diseñadas para comprender y/o transformar un momento concreto de la historia. Por otro lado, los discursos, en particular los hegemónicos dentro de una sociedad, están fuertemente afectados por las relaciones de poder. Pueden emplearse para ocultar rasgos esenciales del mundo de cara a facilitar la explotación, la subordinación o la dominación. La evaluación de los discursos es una labor necesaria si queremos realmente aspirar a una teoría crítica que nos mueva en una dirección emancipadora y que nos ayude en "el autoesclarecimiento por parte del presente de sus luchas y deseos" (Marx, 2014).

En la línea con lo señalado, el objetivo de este artículo es someter a crítica el discurso de la exclusión social. Comenzaremos exponiendo algunos rasgos centrales de nuestro mundo en globalización para, a continuación, exponer las razones que hacen a este discurso profundamente inadecuado. Distrae la atención de características fundamentales de nuestro mundo, de modo que puede ser instrumentalizado para ocultar relaciones que se encuentran a la base de la explotación, la dominación o la subordinación. Frente a este discurso se propondrá otro que podría evitar estos peligros, el de la incorporación adversa.

\section{La globalización}

Bajo el término "globalización" se subsume actualmente un conjunto muy amplio de fenómenos o procesos. Las caracterizaciones que se han ofrecido son muy diversas, así como diversas son las consecuencias que sobre el mundo o sobre nuestra forma de entenderlo se les imputa a tales fenómenos. Diversos autores coinciden en concebir la globalización de un modo muy general y abstracto como un proceso a través del que se está produciendo un aumento de la densidad, la frecuencia, la amplitud, la profundidad y la velocidad de las interconexiones sociales a nivel global (Giddens, 1994, 67-68; Held, McGrew, Goldblatt, y Perraton, 2002, 2; Walby, 2009, 36). Ante esta caracterización, no obstante, rápidamente debemos alertar en contra de la tendencia a entender el "nivel global" como un espacio homogéneo y autónomo, como el nivel propio en el que tuviese lugar la globalización, desconectado de otros espacios como el local o el nacional (Mezzadra y Neilson, 2017, 245). Antes bien, los procesos globales implican un entrelazamiento entre estos niveles, borran los límites entre ellos. El concepto de la globalización, ante todo, nos empuja a rechazar la idea de que los procesos sociales relevantes para los individuos son los que encuentran su lugar exclusivo en el marco nacional (Sassen, 2007, 11), una suposición fundamental dentro del marco conceptual "keynesiano-westfaliano" que dominó el discurso filosófico-político desde el final de la Segunda Guerra Mundial hasta el final del siglo XX (Fraser, 2008, 31-39). En contra de esta suposición, sólo podremos comprender el mundo contemporáneo reconociendo la posibilidad de que lo local sea al mismo tiempo global, de que lo global sólo sea posible a través de su materialización en lo local o en lo nacional, de que lo nacional deba ahora pensarse como algo desnacionalizado o de que los nuevos sitios de la globalización no se definan mediante las fronteras tradicionales (Sassen, 2015, 179-184). Por todo ello, "una 
de las principales características de los procesos de globalización contemporáneos consiste en la continua transformación de las diferentes escalas geográficas cuya estabilidad ya no puede ser dada por hecho" (Mezzadra y Neilson, 2017, 85)

No debemos perder de vista que, aunque no sea el único elemento relevante, sin duda las dinámicas económicas son las que condicionan con más fuerza las características de la actual fase de la globalización. Si bien la globalización, tal como señaló Marx, siempre fue una exigencia de las dinámicas expansionistas del capital (Marx y Engels, 2008, 38-39), las transformaciones estructurales que ha vivido el orden sociopolítico en las últimas décadas ha conferido un nuevo ímpetu a lo económico en el papel de guía de las relaciones y transformaciones globales.

Tampoco hay que olvidar que la fase actual de la globalización se ha producido en el contexto de la conquista de la hegemonía ideológica de un nuevo modo de entender la organización política, el neoliberalismo, el cual "está reconfigurando las relaciones entre los gobiernos y los gobernados, el poder y el conocimiento y la soberanía y la territorialidad" (Ong, 2006, 3). De acuerdo con ese nuevo orden neoliberal, el capital debía verse liberado de los tradicionales impedimentos y controles a los que en el modelo keynesiano-westfaliano el Estado le había sometido; la competencia y el emprendimiento debían convertirse en reglas fundamentales para la constitución, ya no solo del mercado, sino de la identidad de cualquier agente social; el Estado debía privatizar e introducir en el mercado cualquier bien o sector que aún no se encontrase bajo sus leyes; incluso la racionalidad aplicada en el ejercicio de la soberanía estatal debía transformarse a imagen de la gestión económica de la empresa privada, dando origen a una gobernanza por parte de expertos (Brown, 2003; Harvey, 2007, 64-67).

Por todo ello, el papel y la realidad del Estado se han transformado profundamente. No se trata tanto de que el Estado haya perdido poder, sino de que su forma y ámbitos donde ejercerlo se han reconfigurado. La globalización ha ampliado el tipo de actores relevantes en los procesos de toma de decisiones y de implementación de políticas, del mismo modo que ha complejizado el tipo de interacciones posibles. Todo ello sería más adecuadamente descrito como un proceso de coevolución entre una diversidad de entidades que como procesos de erosión o disminución (Walby, 2009, 43). A lo largo de estos procesos, algunos países han ganado más poder mientras que otros lo han perdido, del mismo modo que algunas actividades del Estado han sido fortalecidas mientras que otras se han visto debilitadas (Ong, 2006, 75).

Más decisivo, no obstante, es que la globalización ha abierto nuevos ámbitos para la gestión estatal y ha ampliado el marco respecto al que pueden y deben evaluar las consecuencias de sus decisiones. La globalización ha abierto nuevos espacios políticos y tecnologías de poder heterogéneas y no convencionales (Mezzadra y Neilson, 2017, 225-229; Ong, 2006, 75-77). El Estado no es una víctima de la globalización, sino un cooperador necesario, pues de él depende aún el marco regulatorio de los espacios en los que cualquier fenómeno global debe encontrar asiento (Mezzadra y Neilson, 2017, 234). El espacio de las cuestiones que se abren a la intervención estatal se expande hasta niveles nunca alcanzados anteriormente. La participación en comisiones intergubernamentales y en negociaciones trasnacionales se vuelve una labor cotidiana. Se ponen a su disposición herramientas que trascienden o difuminan los tradicionales límites del espacio nacional (externalización de dispositivos fronterizos y de control, zonas económicas especiales, marcos regulativos y agencias de control y decisión supranacionales, etc.). Por todo ello podría decirse que la 
globalización no limita necesariamente el ámbito de acción de los Estados, sino que, más bien, la expande enormemente (Held et al., 2002, 548). El neoliberalismo, como claramente vio ya Foucault, no se apoya en la reducción de la actividad del Estado, sino que espera de este "una vigilancia, una actividad, una intervención permanente" (Foucault, 2012, 138). Pero esta intervención, eso sí, debe estar guiada por la racionalidad económica, lo que en las condiciones actuales de la globalización significa que los Estados "están forzados a ser flexibles en sus concepciones de la soberanía y la ciudadanía si quieren ser relevantes para los mercados globales" (Ong, 2006, 76).

La globalización señala el reforzamiento de un nivel de integración sistémica por encima del tradicional marco estatal, pero este espacio global está caracterizado por una enorme complejidad y heterogeneidad (Mezzadra y Neilson, 2017, 119). Por un lado, los agentes relevantes a nivel geopolítico se han diversificado. Frente a la antigua concepción de las relaciones internacionales como interacciones sólo entre Estados movidos por la consecución de sus propios intereses, hoy se insiste en un pensamiento trasnacional que pone el foco en las múltiples relaciones establecidas con múltiples fines entre diversos agentes: Estados, individuos, compañías privadas trasnacionales, agencias intergubernamentales, grupos privados de interés, instituciones internacionales, tribunales de justicia supranacionales, etc. (Rodrigo, 2016).

Por otro lado, esta diversificación también se refleja en el funcionamiento del capitalismo global. Frente a la tradicional imagen marxista del obrero industrial como figura paradigmática de la clase trabajadora, la flexibilidad demandada por la globalización neoliberal ha impuesto una multiplicación de los tipos de trabajo, de producción, de consumo, etc., que se traduce en una multiplicación de las posiciones subjetivas y experiencias vitales de los individuos que constituyen la fuerza de trabajo del capitalismo global (Mezzadra y Neilson, 2017, 111-117).

Tales procesos de multiplicación sólo son posibles mediante una diversificación de los estatus legales de las personas y de los marcos regulatorios que se imponen sobre zonas acotadas aún dentro de un mismo Estado (Ong, 2006, 77-79). El espacio político de los Estados tiende a fragmentarse en diversas zonas donde se aplican regulaciones especiales en función de su potencial económico frente a los mercados globales. Tal fragmentación se acompaña por una diversificación de los estatus de los residentes en los Estados, a los que corresponderían diferentes derechos y modos de gestión, de cara a maximizar su potencial productivo según las exigencias del mercado. El ejemplo paradigmático de estos procesos son las zonas económicas especiales (Mezzadra y Neilson, 2017, 254-271; Ong, 2006, 97-118), los enclaves industriales ubicados en espacios fronterizos (Mezzadra y Neilson, 2017, 271-278) o las zonas francas portuarias (id., 240-242). Lo peculiar de estos espacios es su interconexión en redes creando una compleja geografía que no puede comprenderse desde el marco territorial del Estado-nación. Por otro lado, no debería creerse que la diversificación de las redes globales se desarrolla al margen de las particularidades culturales de cada región sobre las que se asientan, como si estuviesen construidas por sujetos "cosmopolitas" más allá de adscripciones particulares. Todo lo contrario, en su configuraciones concretas las redes del mercado global tienden a asumir rasgos etnicizados que refuerzan los estereotipos culturales y de género (Ong, 1999, 3-5; 2006, 122-123). A esta diversificación de los espacios, sistemas regulatorios y agentes que fragmentan internamente el Estado-nación, hay que añadir todo aquel otro conjunto de regulaciones supranacionales que condicionan y se superponen con las regulaciones estatales, así como 
la creciente importancia que están adquiriendo las regulaciones privadas para gestionar diversos ámbitos sociales (Sassen, 1999, 411-414).

La fase actual de la globalización ha traído consigo una profunda transformación y complejización del contexto estructural dentro del que cualquier agente interactúa. Los sistemas normativos se han diversificado. Los marcos dentro de los que encuadrábamos las relaciones sociales se han desestabilizado. Ya no es evidente que el marco prioritario de análisis sea el del Estado o que podamos limitarnos a alguno en particular. Más bien, parece que lo razonable sería presuponer que múltiples dinámicas y escalas de análisis intersecan en cada caso concreto examinado o susceptible de examen. Los agentes involucrados en la toma de decisiones se han heterogeneizado, como también lo han hecho las dinámicas del capital, las formas de trabajo y de producción, así como las subjetividades en las que necesariamente está arraigada toda fuerza de trabajo. Sin embargo, no estamos aquí ante una simple afirmación de la diversidad en sí, sino ante radicales desigualdades de poder.

Sin duda todas estas transformaciones nos deberían empujar a poner en cuarentena los viejos conceptos políticos heredados del pensamiento westfaliano-keynesiano: la unidad del Estado nacional, las nociones de ciudadanía y de soberanía, la correlación entre Estado y sociedad, etc.

\section{El discurso de la exclusión social}

El concepto de exclusión social se emplea para designar al conjunto de "mecanismos mediante los que se impide a individuos y grupos que participen en los intercambios sociales, así como en las prácticas y en los derechos que configuran la integración social y la identidad" (Andersen y Siim, 2004, 6). Este concepto, "implica una división de la población en dos categorías estrictas, situando a los 'excluidos' fuera del funcionamiento de la sociedad, sin los derechos ni las habilidades y recursos necesarios para desempeñar un papel en la colectividad" (Castel, 2007, 34). El discurso de la exclusión tiene su origen en Francia en los años setenta del siglo pasado, en el contexto de los Estados de bienestar europeos (Hickey y Du Toit, 2007, 2). Para los años noventa se había convertido en el discurso dominante en Europa sobre la pobreza y empezaba a generalizarse para contextos no europeos, llegando a eliminar términos tales como "pobreza" o "explotación" de los discursos en torno a los objetivos de las políticas públicas y sobre las causas del empobrecimiento (Du Toit, 2004b, 988; Hickey y Du Toit, 2007, 5; Phillips, 2013, 175). El concepto de la exclusión encuentra su contexto propio de aplicación en "la persistencia de bolsas de pobreza en países ricos y bastante homogéneos" (Du Toit, 2004b , 988), grupos sociales a los que había que responder mediante su inclusión en la normalidad de la nación, en el seguro abrigo de sus esquemas de protección social.

Este discurso, en sus formas más comunes, implica lo que se ha venido denominando un "enfoque residual", según el cual la pobreza es "una consecuencia de haber sido dejado fuera de los procesos de desarrollo, y contiene la suposición de que el desarrollo trae crecimiento y que lo que se requiere es integrar a las personas en los mercados" (Hickey y Du Toit, 2007, 5). La pobreza surgiría entonces en aquellos contextos sociales que han quedado como un residuo no incorporado a los más generales esquemas y procesos de desarrollo. La exclusión de los mercados, de los programas sociales, de los esquemas formales de derechos, de grupos sociales particulares, de bienes sociales específicos, etc., se considera la causa directa de 
la pobreza. Este concepto sirve como etiqueta general bajo la cual muy diversos procesos socio-estructurales son unificados. Bajo esta categorización, se supone que es simplemente su carácter excluyente lo que les conecta casualmente con la pobreza, mientras que la forma y mecanismos específicos de esta conexión se dejan sin analizar.

Ante el problema de la exclusión, la solución está clara: la inclusión (en el mercado, en los proyectos de desarrollo, en las políticas sociales, en la "ciudadanía", en la fuerza de trabajo, etc.). En el contexto actual de la globalización, este enfoque residual ha caracterizado las aproximaciones ortodoxas a la reducción de la pobreza de las instituciones internacionales como el Banco Mundial, "donde el desarrollo es entendido como la expansión y profundización de la globalización, y la ampliación de la participación en esos procesos a través del empleo" (Phillips, 2013, 175). En un mundo global, si la exclusión de sus procesos económicos es la causa de la pobreza crónica global, entonces la respuesta debe ser la inclusión de todos ellos en una globalización que debe ampliarse tanto extensiva (incorporar los espacios residuales que aún quedan fuera) como intensivamente (complejizarse internamente para responder más adecuadamente a la diferencia de los múltiples sujetos crecientemente incluidos). Lo cual no hace sino reproducir la dinámica expansiva del capital global, que ya había sido identificada por Marx (1993, 407-409).

Pues bien, en lo que resta de artículo me centraré en explicar por qué haríamos bien en rechazar este discurso de la exclusión-inclusión si queremos desarrollar una filosofía crítica y emancipadora para el momento actual. El problema no consiste simplemente en la exportación acrítica que ha sufrido este discurso entre diferentes contextos culturales y políticos, desde los debates en torno a políticas públicas de los Estados de bienestar europeos a los debates sobre la pobreza crónica global (Hickey y Du Toit, 2007, 3). El problema, creo, es más de fondo. Se trata de que este discurso de la exclusión-inclusión se basa en una ontología social simplista, completamente inadecuada para abordar las relaciones sociales de un mundo en la actual fase de la globalización. Como veremos, ni para los sujetos paradigmáticamente "excluidos" del mundo global como pueden ser los migrantes irregulares o las personas que caen dentro de lo que se denomina pobreza crónica, este discurso es adecuado, sino que más bien contribuye precisamente a invisibilizar los mecanismos sociales que fundamentan su situación. Ni la exclusión puede pensarse como la causa de sus problemas ni la inclusión puede entenderse como el bien del que carecen. El discurso de la exclusióninclusión se basa en formas de entender el mundo y marcos explicativos y descriptivos, en idealizaciones sobre la sociedad o sobre la ciudadanía, en oposiciones conceptuales binarias y distinciones rígidas, etc. que se han vuelto insostenibles en el mundo contemporáneo.

\subsection{Sobre la idealización de lo político}

Tiene pleno sentido que el discurso de la exclusión-inclusión surgiera en el contexto del Estado de bienestar europeo de los años setenta. En aquella época el pensamiento sociológico y filosófico-político adoptaba lo que se ha denominado "nacionalismo metodológico", el cual puede definirse del siguiente modo:

"El nacionalismo metodológico es una postura en las ciencias sociales que presupone injustificadamente el Estado-nación, lo trata acríticamente como la forma natural de 
organización social y/o lo reifica. Se supone que el Estado-nación controla completamente el espacio geográfico y es tratado como sinónimo de sociedad. El nacionalismo metodológico conduce a un malentendido fundamental de la naturaleza de la realidad social al no reconocer las organizaciones transnacionales, subestatales y supranacionales, y al no tener en cuenta la forma en que las naciones están situadas y constituidas por las fuerzas locales, transnacionales y globales” (Sager, 2016, 43).

El nacionalismo metodológico permitía una fácil y rápida respuesta a la pregunta fundamental: “¿en qué incluir?”. El Estado nacional se correspondía con una sociedad delimitada, culturalmente homogénea y organizada por una estructura sociopolítica cuyos rasgos generales, características y límites no eran problematizados. En este contexto, el discurso de la exclusión-inclusión podía proyectar una "normalidad" social identificada por la sociedad nacional y la participación en las estructuras socio-económicas del Estado de bienestar. En mi opinión, este es el origen de una de las características más notables que operan a su base: "la sociedad dominante se concibe como normal; la exclusión de ella se supone el problema. A veces este grupo excluido puede incluso ser "patologizado' y visto como desviado" (Du Toit, 2004b, 1001). Sin embargo, en el contexto fragmentado y heterogéneo de la globalización tales suposiciones se vuelven insostenibles.

El discurso de la exclusión-inclusión se fundamenta sobre una narrativa moral que presupone acríticamente aquello en lo que incluir. Esta inclusión como regla general es considerada en los discursos hegemónicos sobre desarrollo y pobreza un indiscutible bien social (Mezzadra y Neilson, 2017, 25). Estos se desarrollan "en términos de presuposiciones normativas implícitas sobre cómo debería ser organizada la vida social" (Hickey y Du Toit, 2007, 3). Al asumir un supuesto trasfondo evidente, no problematizado ni problemático en el que incluir, se adopta una posición paternalista y condescendiente hacia los "excluidos" mismos, cuya agencia o papel político en este proceso es negada ante el supuesto básico de que "nosotros" conocemos de partida dónde ellos quieren, pueden y necesitan ser incluidos (Du Toit, 2004a, 29). Seguramente esto podía tener sentido dentro del modo keynesianowestfaliano de entender el mundo y su correspondiente nacionalismo metodológico que aun mayoritariamente se aceptaba dentro de los Estados de bienestar europeos de los años setenta (id.). Pero en el mundo fragmentado, conflictivo y heterogéneo de la globalización, en el que no se puede presuponer entornos sociales y políticos acotados, homogéneos e incontrovertidos en los que incluir, necesitamos un marco conceptual más complejo. El discurso de la exclusión simplifica en exceso la realidad social al basarse en "una concepción inútilmente monolítica y homogénea de la naturaleza de la sociedad" (Du Toit, 2004b, 1005). Por ello, como veremos más adelante, colabora en la ocultación de las complejas relaciones de poder del mundo en globalización.

\subsection{Sobre la fragmentación del espacio de la globalización}

Como hemos visto, la globalización se caracteriza por la multiplicación y diversificación de los espacios normativos, de los procesos y modos de producción de las figuras del trabajo, de las subjetividades relacionadas con ellas, etc. En este contexto de fragmentación, las clásicas nociones de ciudadanía, de homogeneidad nacional de integridad legal 
y exclusividad soberana dentro del territorio nacional, no pueden seguir siendo asumidas acríticamente. Sobre este trasfondo, las preguntas en torno a quién y en qué integrar se vuelven enormemente problemáticas.

La figura del ciudadano-trabajador (asalariado), exclusivamente en torno a la cual giraba la idea del funcionamiento de la sociedad en la época del capitalismo organizado de Estado del período de posguerra (Fraser, 2009, 89-92), empieza a ser definitivamente desarticulada (Mezzadra y Neilson, 2017, 283). Las críticas feministas, según las cuales esta figura servía para ocultar el trabajo de reproducción de la sociedad generalmente realizado por mujeres y privilegiar a los hombres que realizaban el trabajo productivo fuera del ámbito doméstico, se han visto reforzadas por la diversificación, flexibilización y precarización del trabajo que son exigidas por la globalización neoliberal. Ante esta desarticulación, se vuelve problemática la idea de la inclusión a través del trabajo que se presupone en los discursos dominantes de la exclusión-inclusión (Phillips, 2013, 175). Lo relevante serían las cualidades de las relaciones que fundamentan las heterogéneas (y desiguales) formas de trabajo y no la simple inclusión en un mercado en el que el trabajo ya no asegura en muchos casos la subsistencia familiar y menos aún la participación social como ciudadanos iguales.

Pero el nacionalismo metodológico no sólo presuponía una conexión entre el trabajador y el ciudadano. De un modo más general presuponía un isomorfismo entre ciudadanía, soberanía, grupo de solidaridad y nación, desarrollado en un espacio territorial delimitado por las fronteras estatales. Este isomorfismo, en el que todos los miembros debían incluirse, era el que garantizaba el correcto funcionamiento de la sociedad. En este modelo de sociedad, "la traducción es casi uno a uno: la ciudadanía se refleja en el concepto de un sistema jurídico nacional, el soberano en el sistema político, la nación en el sistema cultural y el grupo de solidaridad en el sistema social, siendo todas las fronteras congruentes y definiendo en conjunto la piel que mantiene unido el cuerpo de la sociedad" (Wimmer y Schiller, 2002, 227). El problema es que los desarrollos del actual mundo en globalización hacen que este isomorfismo no sea más que una caricatura insostenible.

El territorio de soberanía estatal como un espacio sometido a una misma estructura normativa, como un espacio homogéneo en el que todos los ciudadanos son incluidos como iguales (o deben serlo si se encuentran "excluidos"), ha entrado también en crisis. La soberanía en la globalización se torna "graduada". El interior del espacio político nacional, que antes se consideraba homogéneo, se fragmenta normativamente (Ong, 2006, 77-78). En un espacio global que no se caracteriza por la homogeneidad sino por una heterogeneidad de enclaves concretos conectados entre sí a modo de nodos de diversas características, la racionalidad gubernamental busca articular su geografía de un modo diferencial según la potencialidad de cada zona ante al mercado global. Las zonas francas portuarias constituyen un ejemplo histórico de esta fragmentación territorial-normativa (Mezzadra y Neilson, 2017, 240-243). Las "zonas económicas especiales" constituyen el ejemplo paradigmático de la forma actual de la globalización (Ong, 2006, 97-118). Si negativamente estas zonas se definen como espacios sobre los que no se aplican ciertas leyes nacionales, Ong insiste en que positivamente estos espacios se diseñan para "promover oportunidades para mejorar la cualificación de los trabajadores, mejorar los servicios sociales y las infraestructuras, experimentar con mayores derechos políticos, etc.” (id., 78). La pregunta aquí es si tiene sentido 
tratar de volver a proyectar ese fondo homogéneo de "normalidad" social en el que todos deben ser incluidos. Este fondo, al menos, no debería presuponerse como no controvertido.

\subsection{Sobre la invisibilización de las dinámicas estructurales de la desigualdad}

Al presuponer que lo malo es la exclusión de los sistemas e intercambios sociales, frente a lo cual la inclusión en los mismos sería la justa respuesta, el discurso de la exclusióninclusión "puede servir para distraer la atención de las dinámicas globales y sistémicas de desigualdad, empobrecimiento y conflicto dentro de esos sistemas sociales más grandes" (Du Toit, 2004b, 1001). En el contexto del incuestionable dominio de la racionalidad economicista dentro de la actual forma neoliberal de la globalización, el discurso de la exclusióninclusión contribuye de dos modos a la justificación y reforzamiento de sus dinámicas. Por un lado, sirve para legitimar la perspectiva de los actores hegemónicos de este tipo de globalización, según la cual la falta de desarrollo y la pobreza se debe precisamente a la exclusión respecto a las fuerzas de mercado globales (Phillips, 2011, 390). El discurso de estos agentes es sencillo: si queremos favorecer el desarrollo y erradicar la pobreza, se debe lograr la inclusión de todos en los procesos de la globalización. ¿Pero qué ocurriría si fuesen estos mismos procesos las causas de la pobreza y de la falta de desarrollo? Es decir, ¿qué ocurriría si estos procesos fuesen en sí mismos explotadores? Pues bien, como se señaló antes, el discurso de la exclusión ha servido precisamente para dejar de lado en los discursos públicos los conceptos de pobreza o explotación.

Un segundo sesgo del discurso de la exclusión-inclusión se manifiesta en su habitual denominación de "exclusión social". Este discurso pretendería atacar los sistemas que impiden el pleno desenvolvimiento de los agentes en el funcionamiento de la sociedad, pero mediante esta denominación se presupone que aquello que se opone a este fin son "el resultado de factores sociales extrínsecos - racismo, cultura, ideología o política - que socavan la participación 'normal' en el funcionamiento de una economía que se considera libre de valores e intrínsecamente neutral" (Du Toit, 2004b, 1002). Ante todo, recordando la cita de Castel, la sociedad es una máquina con esenciales funcionamientos, la exclusión de los cuales se concibe como conceptualmente incompatible con una vida plena en sociedad. Estos funcionamientos son algo neutral y necesario, y los conflictos y problemáticas relaciones de poder que causan su mal funcionamiento responden a una esfera "social" al margen de sus reglas. De acuerdo con Du Toit, esto denota una concepción funcionalista de los procesos sociales y un crudo economicismo (id.). Lo que falta aquí es un análisis del modo en que estos factores sociales, culturales o políticos siempre han estructurado lo económico, y el modo como lo económico en sí mismo está inextricablemente vinculado a las desiguales relaciones de poder y a la violencia.

\subsection{Sobre la insostenible dicotomización entre la exclusión y la inclusión}

Pero en un nivel más general debemos preguntarnos si es adecuado explicarnos la situación de los "pobres globales", de las zonas que no han alcanzado un grado de desarrollo mínimamente aceptable en comparación con otras o, en general, las diversas injusticias que sufren las personas o grupos sociales como si sus causas se encontrasen en su "exclusión" 
o "desconexión" respecto a las dinámicas sistémicas de nuestras sociedades en globalización. Esta explicación, la propia del discurso de la exclusión-inclusión, se basa en una muy deficiente comprensión de estas dinámicas o en su ideológico "blanqueamiento". En contra de lo que este marco de análisis quiere hacer creer, la pobreza, la falta de desarrollo, la desposesión o la "irregularidad" de muchas personas empujadas a migrar no son productos accidentales, externos, de las dinámicas económicas de la globalización, sino sus productos y sus condiciones normales de funcionamiento (Du Toit, 2004b, 1002).

Tal como vimos en la cita de Castel, este discurso se basa en una estricta separación conceptual entre los incluidos y los excluidos, entre el "adentro" y el "afuera" del funcionamiento de la sociedad. Esta categorización desfigura y oculta la compleja causación estructural de los fenómenos sociales y limita la potencialidad de los análisis en el contexto de la globalización (Bracking, 2003, 5). La pobreza o la explotación tienen carácter relacional, es decir, la pobreza o explotación de algunos provee la condición necesaria para la producción de oportunidades privilegiadas para otros (Mosse, 2010, 1156-1159). No podemos individualizar la pobreza, en la línea de la economía neoclásica y los modelos de elección racional, viéndola como disfunciones temporales de una supuesta igualdad política inclusiva y causada simplemente por las malas elecciones o hábitos personales de algunos (ignorando el trasfondo estructural o presuponiéndolo como algo neutral), ni podemos excepcionalizar la pobreza como si fuese algo ajeno al funcionamiento normal de los mercados (Bracking, 2003, 6-7; Mosse, 2010, 1157-1158). Desde la perspectiva de los "excluidos", el discurso que aquí analizamos "tiene poco sentido: ellos están siempre muy incluidos” (Bracking, 2003, 7).

Las condiciones actuales de la globalización imponen la demanda de trabajadores flexibles y baratos. En este contexto, algunos estudiosos de las migraciones insisten que ni siquiera los migrantes "irregulares", otro de los ejemplos paradigmáticos junto a los colectivos sometidos a la pobreza crónica, pueden entenderse adecuadamente como sujetos excluidos. Todo lo contrario, desempeñan un papel esencial en el funcionamiento de las dinámicas económicas globales, del mismo modo que los mecanismos legales y dispositivos fronterizos que determinan su estatus son piezas esenciales "de la producción de la mercancía fuerza de trabajo y del estatuto peculiar de la producción de esta mercancía respecto de las otras" (Mezzadra y Neilson, 2017, 126). La producción legal de ilegalidad, que se supone una de las formas más extremas de "exclusión", responde en realidad a un proceso activo de inclusión que busca la subordinación de su trabajo (De Genova, 2015, 5).

Lo mismo puede decirse del resto de categorías que acríticamente pueblan el espacio del discurso de la exclusión-inclusión, tales como el racismo o el sexismo. Del mismo modo que la ilegalización, "el racismo trata de mantener a la gente en el interior del sistema de trabajo, no expulsarla de él; el sexismo persigue el mismo objetivo" (Wallerstein y Balibar, 1991, 58). Lo que se busca en cada caso es una mano de obra con ciertas características, según relaciones que pueden ser más o menos explotadoras. Todos estos sujetos que el discurso de la exclusióninclusión pinta como marginales, expulsados de la sociedad, en verdad son "protagonistas centrales en el drama de la 'fabricación' del espacio, del tiempo y de la materialidad de lo social mismo" (Mezzadra y Neilson, 2017, 188). Las características de sus situaciones no se deben a su exclusión, sino a los diversos modos en que están incluidos (Du Toit, 2004b, 1002).

Es necesario rechazar la tajante distinción que el discurso que aquí estamos analizando establece entre la exclusión y la inclusión, y la idea de que la primera es un mal que debe 
ser contestado con el incuestionable bien de la inclusión. En realidad, el problema relevante es la inclusión, más concretamente, los modos en los que esta inclusión se realiza, las posiciones desventajosas y los procesos de explotación, expropiación, subordinación y/o dominación en los que las dinámicas estructurales de nuestro mundo en globalización sitúan a algunos en beneficio de otros.

Esta forma de platear estos problemas se inscribe dentro de una tendencia más general que ha tomado fuerza a raíz de los complejos procesos de la globalización y la puesta en cuestión del marco conceptual filosófico-político clásico. Me refiero a la puesta en cuestión de la forma binaria de entender las categorías, separadas por una línea claramente definida. La oposición entre exclusión e inclusión debe ser rechazada. Lo mismo se ha dicho de pares conceptuales que habían sido fundamentales en el pensamiento hasta el momento actual: trabajo libre y esclavo, trabajo productivo e improductivo, interior y exterior de una comunidad política supuestamente unificada, residentes temporales y permanentes, mercados formales e informales, etc. El desafío teórico en este contexto es desarrollar una forma más crítica de conceptualizar los límites, de diseñar unos marcos teóricos mejor capacitados para abordar las "zonas grises” que constituyen en realidad nuestros espacios sociales (Balibar, 2015, 73).

\section{La incorporación adversa}

La expresión "incorporación adversa" ha tomado fuerza dentro de los análisis de la pobreza como una perspectiva más adecuada que el discurso de la exclusión-inclusión, al basarse en una ontología social más crítica y poder así evitar sus problemas antes mencionados. La situación de los pobres globales y, en general, la de cualquier agente de un grupo o clase social, sólo puede comprenderse en relación con el resto de posiciones sociales. Frente al "enfoque residual" que caracteriza al discurso de la exclusión, el de la incorporación adversa se basa en un "enfoque relacional" (Phillips, 2013, 175). Este enfoque "construye una explicación basada en los términos en los que las personas son incorporadas en procesos y estructuras económicas y sociales particulares" (id.). La pobreza no es debida a la marginalidad respecto a las relaciones económicas y políticas desarrolladas históricamente, sino todo lo contrario, como consecuencia de su inclusión en estas relaciones (Mosse, 2010, 1157), más exactamente, por los términos y condiciones de la incorporación (Du Toit, 2004b, 1003). Para terminar con ella, por lo tanto, no se requiere la "inclusión" en tales procesos ni en ninguna supuesta "normalidad" social, pues los agentes explotados, subordinados y dominados ya están plenamente inmersos en ellos, sino la transformación de las relaciones sociales y políticas (Bebbington, 2007, 793). Frente a la absolución que el discurso de la exclusión-inclusión promueve del trasfondo estructural, la perspectiva relacional evidencia la necesidad de poner el foco de análisis sobre los modos en los que se piensa y reproduce la pobreza (id., 796-798). Por todo ello, se propone la idea de "incorporación adversa" como más apropiada (Murray, 2001, 5).

Las relaciones sociales en las que todo agente está inmerso deben abordarse como algo multidimensional, pues no solo implican el modo como las personas están incluidas en el mercado, sino también en el Estado, en la sociedad civil, en la comunidad o en el entorno doméstico (Hickey y Du Toit, 2007,4). El modo en que la sociedad es categorizada a través 
de los discursos hegemónicos, haciendo pensable y justificable la existencia de la pobreza $\mathrm{u}$ otras injusticias, es también un objeto fundamental que debe ser analizado (Bebbington, 2007, 805-807; Mosse, 2010, 1169-1171). Esta perspectiva tiene también efectos sobre el modo en que toda política pública que intente resolver problemas sociales debe ser diseñada, guiada e implementada. Debe estar basada en profundos análisis sociales que no sólo contemplen la economía política, sino también la historia local, la cultura, el reparto del poder político y las relaciones basadas en el género o en cualquier otro rasgo identitario en contextos concretos (Murray, 2001, 5).

Este trasfondo estructural que incorpora adversamente a algunas personas, sometiéndolas a relaciones de explotación, subordinación o dominación, opera no tanto impidiéndoles la entrada, sino impidiéndoles la salida (Phillips, 2013, 178). En nuestro mundo en globalización es necesario pensar en profundidad las "cadenas que atan" a los heterogéneos agentes a sus complejas relaciones laborales, comunitarias, políticas, identitarias, familiares, etc. (Mezzadra y Neilson, 2017, 147). Esto conlleva la necesidad de incorporar en el análisis a agentes de muchos tipos, sistemas normativos de diferentes niveles, dispositivos gubernamentales diversos, etc. Se requiere una exploración paciente de la multiplicidad de vínculos y agentes que constituyen el paisaje social de un mundo en globalización. Esta exploración es además fundamental si se quieren encontrar puntos de conexión, posibles bases de solidaridad para la reconstrucción de una política colectiva emancipadora (Mezzadra y Neilson, 2017, 148).

Emplear aquí la expresión "incorporación adversa" responde a una selección consciente de los términos. Diferentes autores han propuesto términos similares para referirse a parecidas perspectivas de análisis, pero los términos elegidos indican también pequeños cambios en los marcos teóricos o intenciones. Mezzadra y Neilson, por ejemplo, emplean la expresión "inclusión diferencial" (Mezzadra y Neilson, 2017, 186-196). "Adverso" y "diferencial" señalan circunstancias completamente heterogéneas. El segundo denota simplemente que algo es distinto a otra cosa, sin entrar a valorar tal diferencia. En este sentido, es descriptivamente neutral. El primero señala una relación de cierto tipo en la que uno o algunos de los elementos se colocan en una posición desfavorable frente a otro u otros. Es este tipo de relaciones precisamente el que se pretende clarificar mediante la expresión que hemos estado analizando, por lo que el término "adverso" parece más apropiado para el caso. "Incorporación adversa" no pretende describir un estado final, sino evaluar procesos por los cuales los agentes son sistemáticamente explotados, dominados o subordinados. Estos procesos no producen simplemente diversidad, sino que producen injusticia. Estas diferencias de perspectiva también se manifiestan en el empleo que los autores citados hacen de esta expresión, pues se sirven de ella para describir la labor de filtrado que realizan las fronteras estatales para adaptar la entrada de mano de obra a las exigencias del mercado nacional de trabajo. Los términos "inclusión" e "incorporación" indican diferencias similares, pues mientras que el primero denota la acción de poner algo simplemente dentro de los límites de un conjunto, reproduciendo la oposición simple entre inclusión y exclusión, el segundo adopta una perspectiva más propiamente relacional, al denotar el modo en que una persona o cosa está en relación con otras con las que forma un todo.

Problemas terminológicos similares pueden señalarse en relación con la expresión "los pobres globales": 
“Hablar de 'los pobres' es, después de todo, considerar a las personas en cuestión como víctimas pasivas en lugar de agentes y actores políticos potenciales. Es también verlos de manera desconectada, descontextualizada, en abstracción de las relaciones y procesos sociales que han generado su pobreza. Nombrar su difícil situación como 'pobreza', finalmente, es sugerir que ellos simplemente, inexplicablemente, carecen de los medios de subsistencia, cuando en realidad han sido privados de esos medios" (Fraser, 2010, 369).

De ahí que Fraser proponga sustituir el término "los pobres" por "el precariado", que a diferencia de la descripción de estados finales enfatiza los procesos que generan la injusticia y la agencia de los que la sufren, dando cabida además a diversos grados de inclusión/exclusión. El término "global" sería también problemático, pues, como se señaló en la primera parte del artículo, este no es el único marco que tiene relevancia en la causación estructural de la injusticia. Se ha de hablar más bien de la intersección de múltiples marcos (local, nacional, territorial, etc.). Por todo ello, Fraser concluye que lo más adecuado sería emplear la expresión "el precariado trasnacional" en lugar de "los pobres globales" (id., 370).

Frecuentemente se presentan las categorías que organizan lo social como pares binarios en oposición, clara y radicalmente distintos: varón/mujer, ciudadano/extranjero, nosotros/ ellos, etc. (Tilly, 1999). Este pensamiento obsesionado con trazar una distinción tajante entre un adentro y un afuera es precisamente la forma de conceptualizar los límites y de cartografiar lo social que aquí se ha defendido que debemos superar, si queremos adoptar una perspectiva más crítica y capaz de abordar la multiplicidad de vínculos que vemos tomar forma en la fase actual de la globalización.

\section{Conclusión}

Guiados por la idea de que es fundamental analizar los efectos de los discursos en la sociedad, lo que estos visibilizan y ocultan, las justificaciones que facilitan y las políticas públicas que promueven, en este artículo se ha analizado el papel del discurso de la exclusión-inclusión en la crítica de las injusticias. Frente a él, se ha propuesto la noción de incorporación adversa como una perspectiva más adecuada para abordar la complejidad de las relaciones sociales en un mundo en globalización.

Otros discursos empleados frecuentemente para abordar la situación del "precariado trasnacional" $-\mathrm{u}$ otros agentes sometidos a diferentes tipos de injusticias - pueden también ser evaluados desde la perspectiva derivada de la discusión precedente. Este sería el caso, por ejemplo, de los análisis basados en la idea de "vulnerabilidad". Frente a la tendencia que estos análisis muestran de enfocarse "estrechamente en la sensibilidad o resiliencia de los sistemas de subsistencia en relación con conmociones y peligros", es necesario resaltar "la medida en que las relaciones estructurales más amplias que sustentan estos medios de vida pueden crear sistemáticamente pobreza y vulnerabilidad en primer lugar" (Hickey y Du Toit, 2007, 8). Esta perspectiva ayuda a evitar la individualización o la excepcionalización de la vulnerabilidad, la tendencia a explicarla mediante el recurso a supuestas características de las personas o como efectos excepcionales debidos simple- 
mente a malas elecciones de los agentes en el contexto de una estructura neutral. No es la exclusión, la mala suerte o la inevitable exposición al riesgo lo que causa la mayor pobreza o vulnerabilidad de algunos, sino su integración en relaciones sociales explotadoras y precarias, en un contexto en el que la incorporación adversa es fundamental a los procesos sociales de nuestro mundo en globalización.

Frente al típico discurso liberal sobre la justicia centrado en la defensa del reconocimiento o no de ciertos derechos formales, la perspectiva aquí defendida se muestra escéptica ante tal forma de discurrir teóricamente. Esta, en el mejor de los casos es de escasa penetración en la causación estructural y, en el peor, puede ser instrumentalizada para desviar la crítica hacia discusiones retóricas y alejar su atención de las complejas relaciones de poder que constituyen el mundo social. Como se ha indicado anteriormente, la perspectiva de la incorporación adversa insiste en la necesidad de atender a los contextos concretos, de analizarlos pacientemente para comprender las múltiples dinámicas de poder que constituyen las relaciones sociales implicadas específicamente en cada situación de injusticia. Para avanzar hacia la justicia no existen recetas mágicas. Haciéndonos eco de la clásica crítica marxista debemos dar un paso más allá de la esfera formalista liberal y entrar en la espesa textura de las relaciones sociales concretas (que también, por supuesto, incluyen análisis de las leyes y normas formales e informales).

El discurso de la incorporación adversa, finalmente, nos empuja a pensar la sociedad con unas herramientas conceptuales más complejas que aquéllas que postulan límites rígidos y binarios entre pares de categorías, basados en la exigencia de trazar una clara distinción entre el "adentro" y el "afuera". Pensar la "zona gris" dentro de la que se despliega la sociedad es una tarea fundamental si queremos desarrollar un pensamiento crítico útil en un mundo en globalización.

\section{Referencias}

Andersen, J. y Siim, B. (2004), "Introduction: The Politics of Inclusion and Empowerment. Gender, Class and Citizenship", en: Andersen, J. y Siim, B. (Eds.): Politics of Inclusion and Empowerment: Gender, Class and Citizenship, Gordonsville: Palgrave Macmillan, pp. 1-18.

Balibar, É. (2015), Citizenship, Cambridge: Polity Press.

Bebbington, A. (2007), "Social Movements and the Politicization of Chronic Poverty", Development and Change, 38(5), pp. 793-818.

Bracking, S. (2003), "The Political Economy of Chronic Poverty", Chronic Poverty Research Centre. Working Paper(23).

Brown, W. (2003), "Neo-liberalism and the End of Liberal Democracy", Theory and Event, $7(1)$, pp. 1-29.

Castel, R. (2007), La Discrimination négative: citoyens ou indigènes? , Paris: Éditions du Seuil.

De Genova, N. (2015), "Border Struggles in the Migrant Metropolis", Nordic Journal of Migration Research, 5(1), pp. 3-10. 
Du Toit, A. (2004a), "Forgotten by the highway: Globalisation, adverse incorporation and chronic poverty in a commercial farming district of South Africa", CPRC Working Paper(49).

Du Toit, A. (2004b), "'Social Exclusion' Discourse and Chronic Poverty: A South African Case Study", Development and Change, 35(5), pp. 987-1010.

Foucault, M. (2012), Nacimiento de la biopolítica, Madrid: Akal.

Fraser, N. (2008), Escalas de justicia, Barcelona: Herder.

Fraser, N. (2009), "El feminismo, el capitalismo y la astucia de la historia", New left review, 56, pp. 87-104.

Fraser, N. (2010), "Injustice at Intersecting Scales: on "Social Exclusion" and the "Global Poor"”, European Journal of Social Theory, 13(3), pp. 363-371.

Giddens, A. (1994), Consecuencias de la modernidad, Madrid: Alianza.

Harvey, D. (2007), A Brief History of Neoliberalism, Oxford: Oxford University Press.

Held, D., McGrew, A., Goldblatt, D. y Perraton, J. (2002), Transformaciones globales. Política, economía y cultura, Ciudad de México: Oxford University Press.

Hickey, S. y Du Toit, A. (2007), Adverse incorporation, social exclusion and chronic poverty, Chronic Poverty Research Centre. Working Paper.

Marx, K. (1993), Grundrisse. Foundations of the Critique of Political Economy, Londres: Penguin.

Marx, K. (2014), Carta a Arnold Ruge, en Marxists Internet Archive https://www.marxists. org/espanol/m-e/cartas/m09-43.htm

Marx, K. y Engels, F. (2008), The communist manifesto, Londres: Pluto Press.

Mezzadra, S. y Neilson, B. (2017), La frontera como método, Madrid: Traficantes de sueños.

Mosse, D. (2010), "A Relational Approach to Durable Poverty, Inequality and Power", Journal of Development Studies, 46(7), pp. 1156-1178.

Murray, C. (2001), Livelihoods research: some conceptual and methodological issues, Chronic Poverty Research Centre. Background Paper.

Ong, A. (1999), Flexible Citizenship, Durham y Londres: Duke University Press.

Ong, A. (2006), Neoliberalism as Exception. Mutations in Citizenship and sovereignty, Durham y Londres: Duke University Press.

Phillips, N. (2011), "Informality, global production networks and the dynamics of 'adverse incorporation"”, Global Networks, 11(3), pp. 380-397.

Phillips, N. (2013), "Unfree labour and adverse incorporation in the global economy: comparative perspectives on Brazil and India", Economy and Society, 42(2), pp. 171-196.

Rodrigo, Á. J. (2016), "Entre Westfalia y Worldfalia: la comunidad internacional como comunidad social, política y jurídica”, en: García Segura, C. (Ed.), La tensión cosmopolita. Avances y límites en la institucionalización del cosmopolitismo, Madrid: Tecnos, pp. 23-63.

Sager, A. (2016), "Methodological Nationalism, Migration and Political Theory", Political Studies, 64(1), pp. 42-59.

Sassen, S. (1999), "Making the global economy run: the role of national states and private agents", UNESCO(161/1999). 
Sassen, S. (2007), Una sociología de la globalización, Buenos Aires: Katz.

Sassen, S. (2015), "From national borders to embedded borderings", en: Weber, L. (Ed.), Rethinking border control for a globalizing world. A preferred future, Londres y Nueva York: Routledge, pp. 179-189.

Tilly, C. (1999), Durable Inequality, Berkeley, Los Angeles y Londres: University of California Press.

Walby, S. (2009), Globalization \& Inequalities. Complexity and Contested Modernities, Londres: SAGE.

Wallerstein, I. y Balibar, É. (1991), Raza, nación y clase, Madrid: Lepala.

Wimmer, A. y Schiller, N. G. (2002), "Methodological nationalism and the study of migration”, European Journal of Sociology, 43(2), pp. 217-240. 